\title{
A INSERÇÃO DO MOVIMENTO DA MATEMÁTICA MODERNA NA UFRN ${ }^{1}$
}

\section{La inserción de la matemática moderna en la UFRN}

\author{
Arlete de Jesus Brito ${ }^{2}$ \\ Sabrina Susan Lucena Cruz ${ }^{3}$ \\ Josefa Poliana Clementino Ferreira ${ }^{3}$
}

\section{Resumo}

Este artigo apresenta alguns resultados da pesquisa que estamos desenvolvendo em Natal acerca do Movimento da Matemática Moderna nesta cidade. Analisamos a inserção do Movimento da Matemática Moderna na Universidade do Rio Grande do Norte, na década de 1960. Para tal, iniciamos com uma contextualização do Movimento da Matemática Moderna de modo a ressaltar suas relações com os eventos sociais da época e, a seguir, analisamos a importância do convênio firmado entre SUDENE e UFRN para a inserção do MMM nesta universidade.

Palavras-chave: MMM, Instituto de Matemática, SUDENE.

\section{Resumem}

Este artigo trae algunos resultados de una investigación que estamos realizando en Natal a cerca del Movimiento de la Matemática Moderna en la Universidad Federal del Rio Grande do Norte, Brasil, en la década de 1960. Nosotros iniciamos con un estudio del contexto de la época e después analizamos la importancia del convenio entre SUDENE e UFRN para la inserción del MMM en esta universidad.

Palabras-clave: MMM, Instituto de matemáticas, SUDENE.

1 Pesquisa financiada pelo CNPq.

2 Professora do Departamento de Matemática da Universidade Federal do Rio Grande do Norte e dos PPGEd e PPGECNM da mesma universidade. arlete@ccet.ufrn.br

3 Alunas do curso de Licenciatura em Matemática da UFRN: Josefa Poliana Clementino Ferreira poliana.mat@bol.com.br// Sabrina Susan Lucena Cruz. sabrina1susan@yahoo.com.br 
Durante os primeiros anos do século XX, houve várias tentativas isoladas de se iniciar uma reforma no ensino de Matemática. Essa reforma tinha como propósito principal superar a defasagem existente entre o ensino das escolas secundárias e as necessidades técnicas mais recentes, necessidades estas advindas do desenvolvimento industrial e dos novos modos de produção capitalista. Segundo Soares (2001, p. 26), tais reformas isoladas passaram a serem organizadas quando foi instituída em 1908, em Roma, a Commission Internationale de L'Enseignement Mathématique (CIEM) também conhecida pela sigla IMUK de Internationale Mathematische Unterrichts Kommission. Daí surgiu o primeiro Movimento Modernizador, cujo maior defensor foi Felix Kline.

No Brasil, o primeiro movimento modernizador esteve presente, inicialmente, no colégio Pedro II, principalmente devido à atuação de Euclides Roxo. Com a Reforma Campos, em 1931, Euclides Roxo foi convidado a elaborar uma proposta nacional para o ensino de matemática da escola secundária. A proposta elaborada por ele foi inspirada no projeto de Felix Klein e pressupunha, entre outras coisas, que o conceito de função fosse o articulador dos conceitos matemáticos, que o ensino de geometria iniciasse com explorações intuitivas, de modo que esta fosse paulatinamente formalizada e que se inserisse cálculo diferencial na escola secundária. Porém, tal proposta nunca chegou realmente a se efetivar (VALENTE, 2004).

A partir da década de 1950, iniciou-se outro movimento modernizador conhecido como Movimento da Matemática Moderna, embasado na matemática desenvolvida há pouco tempo pelo grupo Bourbarki. Foram vários os motivos que levaram à inserção do MMM nos currículos escolares de vários países. Tal inserção foi justificada pela suposta falta de interesse pela matemática, por parte dos estudantes e pela dificuldade deles em aprender tal conhecimento, porém, segundo Zuñiga (1966, p. 10), o início da reforma se deu com lançamento do satélite russo Sputnik pelos soviéticos, em 1957. Este fato fez com que o bloco capitalista acreditasse que estava em desvantagem científica e tecnológica em relação aos socialistas. Assim, o bloco capitalista, liderado pelos EUA, buscou uma reforma de ensino que possibilitasse ao estudante entrar em contato, o mais cedo possível, em sua escolaridade, com o que houvesse de mais avançado no campo científico.

Conforme Kline (1976), nesta época, o governo norte-americano passou a investir financeiramente na mudança do ensino de matemática. "Talvez seja coincidência, mas nessa ocasião muitos outros grupos decidiram entrar no negócio de criar um novo currículo" (KLINE, 1976, p. 33). Além disso, o MMM tornou-se um baluarte do desenvolvimento científico e tecnológico do capitalismo, desenvolvimento este que, supostamente, levaria a uma sociedade mais "evoluída". É neste contexto que se situa a inserção do movimento da matemática moderna no Brasil, e em particular, no Rio Grande do Norte. 
Não é possível determinar o momento exato em que este movimento chegou ao Brasil, porém podemos indicar alguns caminhos pelos quais o MMM foi inserido nas escolas brasileiras. Um primeiro foi o ensino de álgebra moderna nas universidades, a partir de 1945. A criação do IMPA, Instituto de Matemática Pura e Aplicada, com sede na cidade do Rio de Janeiro, também teve importância naquela inserção. Além disso, a participação dos professores brasileiros Omar Catunda, Alfredo Pereira Gomes e Leopoldo Nachbin na Primeira Conferência Interamericana sobre Educação Matemática em Bogotá, na Colômbia, no ano de 1961, também deve ser destacada, pois tal Conferência, realizada com o apoio da OEA e da UNESCO, teve por objetivo explorar métodos para o ensino de Matemática no nível secundário e universitário e ainda aprovar resoluções com vistas a um projeto de cooperação futura, na área educacional, entre os países participantes. Segundo Soares (2001), os temas abordados na conferência tratavam das novas tendências de um ensino moderno de Matemática.

Outro fator que colaborou para a divulgação do MMM no Brasil foi a criação de grupos para a divulgação dos ideais do MMM entre professores, como, por exemplo, o Grupo de Estudos do Ensino da Matemática (GEEM), que promoveu cursos sobre Matemática Moderna para professores de matemática das escolas secundárias, com o objetivo de superar as dificuldades na aprendizagem deste campo do saber neste nível de ensino, dificuldades estas apontadas em todos os Congressos Nacionais de Ensino de Matemática realizados até então.

No entanto, no Rio Grande do Norte, a inserção do MMM no ensino se fez por outros caminhos. Segundo reportagem do jornal Tribuna do Norte (15/10/1967, p. 7) o MMM começou a ser ensinado na escola secundária por volta de 1966 e, segundo o entrevistado, "a matemática moderna, em Natal, ainda está atrasada. Somente três professores a ensinam: Teófilo Canan, Josafá Cordeira e José Ivaldo. Os estabelecimentos - Colégio Estadual do Atheneu e Colégio Sete de Setembro. Grande número de professores, do método tradicional, se recusa a dar a nova matemática".

Além disso, até aquele ano, o Movimento da Matemática Moderna não fazia parte dos programas das disciplinas da Universidade do Rio Grande do Norte ${ }^{4}$, conforme nos indicam os documentos da época. Nos anos de 1966 e 1967, o exame para admissão da Escola de Engenharia denominado Concurso de Habilitação era composto pelas provas de Matemática I (álgebra e geometria analítica), Matemática II (geometria e trigonometria), Física, Química e Desenho Geométrico. Em ambos os anos, foram exigidos os seguintes conteúdos em matemática:

4 Esta Universidade foi denominada Federal do Rio Grande do Norte somente a partir de 1968. 


\section{Primeira parte: álgebra e geometria analítica.}

Teoria dos erros. Primitivas. Função linear. Distância entre dois pontos. Logaritmos. Determinantes. Parâmetro linear. Área do triângulo. Progressões. Análise combinatória. Linha reta. Circunferência. Teoria das equações. Estudo das funções. Ponto que divide segmentos numa razão dada. Forma simétrica da equação da reta. Bissetriz. Propriedades da derivada. Máximos e mínimos. Curvas do segundo grau. Interseção de retas. Equação Paramétrica da reta. Interseção de retas e curvas. Polinômios. Circunferência. Derivadas. Teoria. Distância entre dois pontos. Interseção de retas e círculos.

\section{Segunda parte: geometria e trigonometria.}

Retas e planos. Resolução de triângulos obliqüângulos. Corpos redondos usuais. Áreas e volumes. Equações trigonométricas. Superfícies e poliedros em geral. Resolução de triângulos retângulos. Seções cônicas. Transformações. Multiplicação e divisão de arcos. Soma e subtração de arcos. Vetores. Projeção. Esfera. Pirâmide. Funções trigonométricas. Funções lineares. Sistemas de equações. Esfera e superfície cônica. Fórmula de Moivre. Perpendiculares e Planos.

Como observamos, teoria dos conjuntos e estruturas algébricas não faziam parte dos conteúdos solicitados para admissão na Escola de Engenharia. Além disso, tais teorias também não faziam parte do programa da disciplina de Cálculo Infinitesimal I daquela Escola, conforme podemos observar pelas cópias dos documentos abaixo:
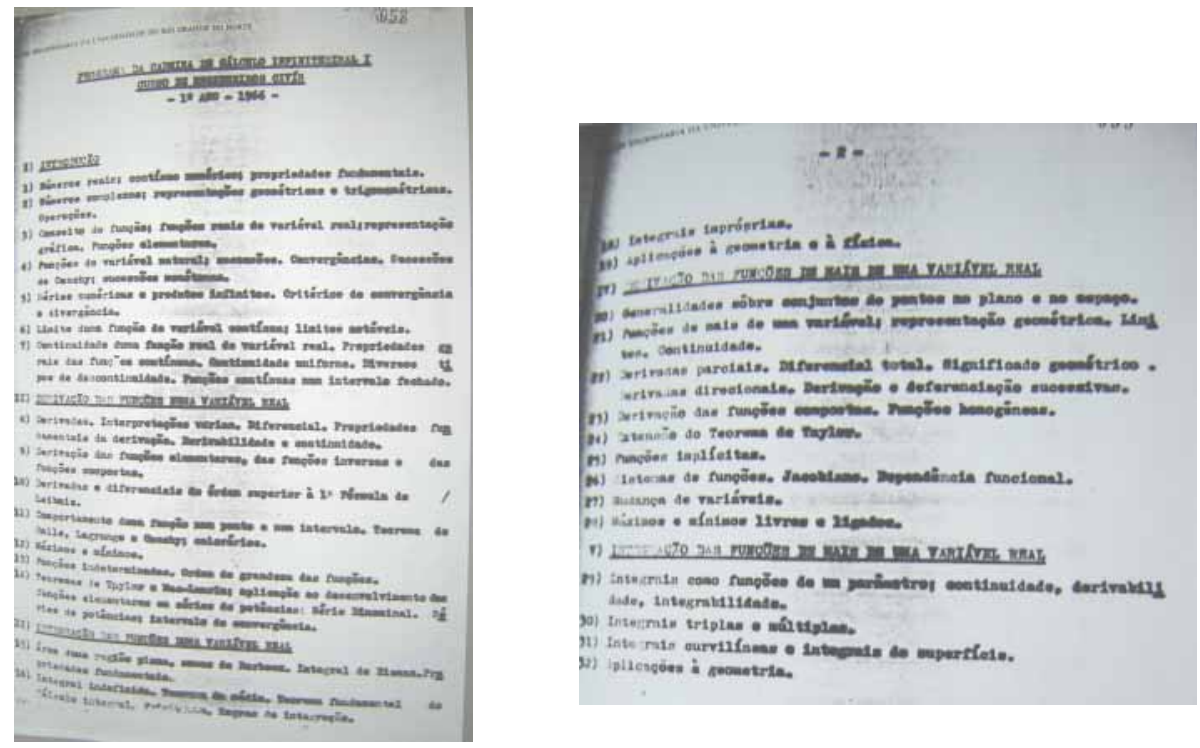
Apesar disso, localizamos o diário de classe de um professor, não identificado, segundo o qual, em 12/09/1966, teria abordado propriedades dos conjuntos em sua aula e uma avaliação (figura abaixo) que solicita a determinação de ponto de acumulação de um conjunto.

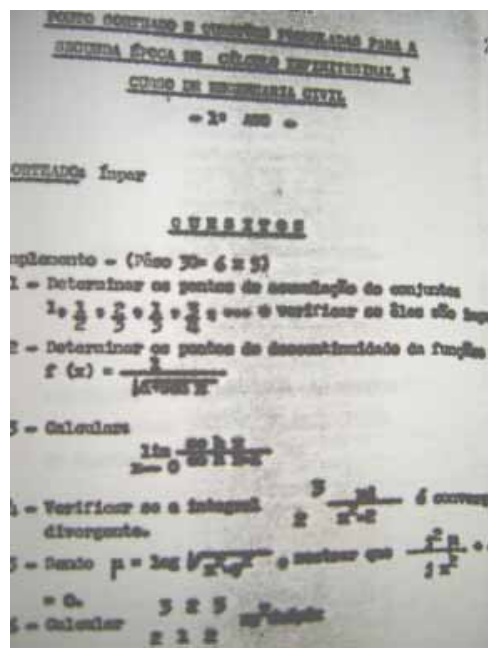

Porém, no ano de 1967, teoria dos conjuntos já fazia parte do programa de Cálculo Infinitesimal I, como podemos observar abaixo:

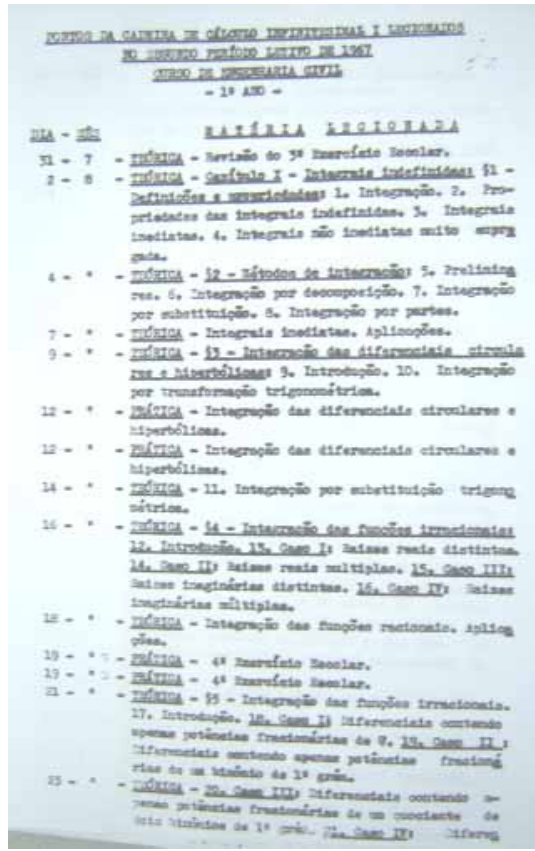

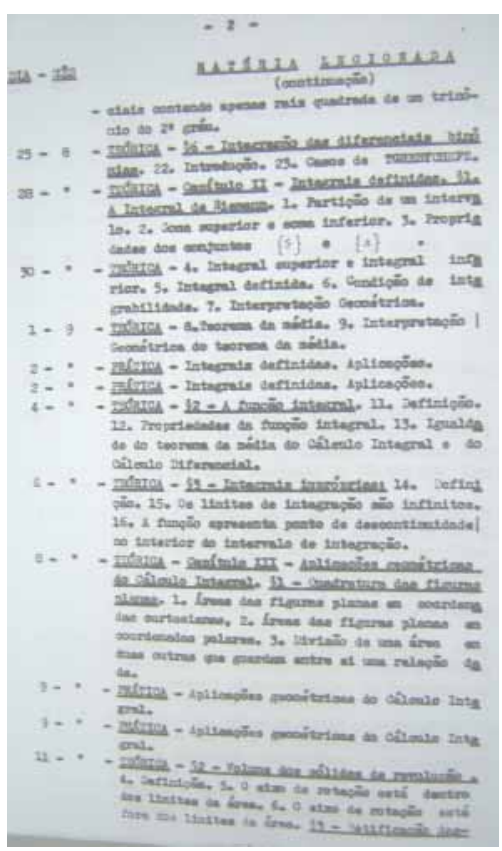


Teoria dos conjuntos passou a fazer parte oficialmente do curso de cálculo em 1967, porém, em dezembro de 1965, o Centro de Ensino de Ciências no Nordeste (CECINE) encaminhou para a UFRN informações e ficha de inscrição para o curso de verão de Matemática que ocorreria em fevereiro de 1966. Tal curso teria lugar em Salvador, Bahia, e propunha-se a abordar tópicos de Matemática Moderna. Porém, não houve, neste curso, candidatos da Universidade do Rio Grande do Norte, até porque naquela época ainda não existia na UFRN, um Instituto de Matemática e os professores da Escola de Engenharia estavam muito mais preocupados em realizar aprofundamentos em assuntos relativos à matemática aplicada, como podemos observar pelos relatórios daquela Escola, escritos entre os anos de 1964 e 1966. A criação do Instituto de Matemática foi determinante para a inserção do MMM na Universidade do Rio Grande do Norte, conforme veremos a seguir.

O Instituto de Matemática (IMURN) foi criado oficialmente em 1966 e sua direção ficou a cargo do professor Dirceu Victor Gomes de Holanda. Porém, apenas em 1968, com o decreto 62091 (09/01/68), que determinava a nova estrutura da UFRN, tal Instituto se concretizou, pois até então funcionava com professores e estrutura administrativa da escola de engenharia. Ainda neste ano, o curso de Licenciatura em matemática que, desde 1966 pertencia à Faculdade de Filosofia, Ciências e Letras de Natal, passou a fazer parte do IMURN.

No projeto de criação do IMURN encontram-se alguns objetivos a serem alcançados por aquele instituto, entre os quais podemos destacar os seguintes: aperfeiçoar o pessoal docente, no setor de matemática, por meio de cursos de Análise Matemática e de Álgebra Moderna e elaborar e ministrar o "Curso de Iniciação à Matemática" (CIM), destinado a prováveis futuros universitários, egressos do então ciclo ginasial.

Estes objetivos relacionam-se à inserção do Movimento da Matemática Moderna na UFRN. Eles se efetivaram graças a um convênio denominado Convênio de Ensino Técnico de Curso Superior, firmado em março de 1966, entre a UFRN e a Superintendência de Desenvolvimento do Nordeste (SUDE$\mathrm{NE}$ ).

A SUDENE foi criada em 1959 como uma agência de desenvolvimento regional incumbida de planejar e coordenar os programas socioeconômicos de interesse do Nordeste. Entre eles, alguns relativos à educação, conforme lei 4869 de 01/12/1965, artigo 28: "Ficam acrescidas ao art. 5.', da Lei n. ${ }^{\circ}$ 4.239, de 27 de junho de 1963, as seguintes alíneas: a) financiamento, total ou parcial, de programas ou projetos que visem à melhoria do sistema de ensino técnico profissional”. Tal parágrafo possibilitou o convênio supracitado que previa: 
Pagamento de dois (2) professores visitantes, atendendo à continuidade do Programa em execução e constante de plano de aplicação de outro convênio SUDENE/UFRN, de aperfeiçoamento de pessoal docente no setor de Matemática. Os citados professores vêm dando dois cursos um (1) de Análise Matemática e outro de Álgebra Moderna desde janeiro último.

Pagamento de oito (8) alunos-bolsistas (sendo quatro da Escola de Engenharia - setor de Física e quatro do Instituto de Matemática) visando a um preparo especializado nesses dois setores básicos. Os alunos-bolsistas do Instituto de Matemática terão algumas tarefas docentes no 'Curso de Iniciação à Matemática'.

Para dar continuidade ao programa do Instituto de Matemática, pretende-se adquirir o material bibliográfico estritamente necessário ao atendimento das necessidades imediatas. (SUDENE/UFRN, 1966, p. 1 - 3).

O aperfeiçoamento do pessoal docente referido neste convênio ocorreu com os cursos de Álgebra Moderna e o de Análise Matemática, ministrados, respectivamente, pelos professores Antonio Mario Sette, da Universidade Federal de Pernambuco e Nelson de Almeida Braga do Instituto de Matemática da Universidade Federal do Ceará. Vale salientar o papel de difusor das idéias do Movimento da Matemática Moderna, exercido pela Universidade Federal de Pernambuco ${ }^{5}$, conforme podemos observar pela seguinte passagem escrita em 1962, por Marshall Stone ${ }^{6}$, professor da Universidade de Chicago e então presidente do ICMI (International Committee of Mathematical Instruction):

A imensidade do Brasil, a sua diversidade, e a autonomia dos Estados, segundo a nova lei referente ao ensino secundário, levam a uma concentração do esforço para uma reforma num pequeno número de estados - por exemplo, na Guanabara (visto que o Rio de Janeiro continua a ser capital cultural senão política do país), em São Paulo (porque é o estado industrial por excelência e dispõe de maiores meios financeiros que os outros), e em Pernambuco (porque é o mais forte estado de uma região típica no que diz respeito aos problemas do desenvolvimento econômico e social do país). O êxito em dois ou três estados duma reforma fundamental do ensino matemático secundário facilitaria muito a adaptação desta reforma às necessidades e às condições dos outros estados (STONE, 1962, p.19, apud SOARES, 2001).

Temos poucas informações sobre o MMM na Universidade Federal do Ceará.

6 Stone foi presidente deste comitê entre os anos de 1961 e 1967, período de disseminação dos ideais do MMM nos países do bloco capitalista. 
Deste modo, observamos que o convênio firmado entre a SUDENE e a UFRN, e o conseqüente pagamento do curso ministrado pelo professor Sette inseriu-se em uma estratégia, elaborada em 1962, de divulgação das idéias do Movimento da Matemática Moderna em nosso país, estratégia esta que esteve desde seu início atrelada ao aspecto econômico como critério de escolha dos Estados que participariam primeiramente desta reforma e que seriam os disseminadores dela.

Entre os participantes do curso de Álgebra Moderna estavam os alunos bolsistas do convênio SUDENE/UFRN, que em agosto de 1966 começaram a ministrar o CIM. Segundo o boletim Notícias do Instituto de Matemática, o Curso de Iniciação à Matemática iniciou-se em 12 de agosto de 1966, era destinado a alunos do $2 .^{\circ}$ ciclo secundário e visava a:

dar uma preparação básica em Matemática Moderna, indispensável aos jovens que pretendem ingressar com êxito em qualquer curso de nível universitário. Registrou-se uma matrícula de 122 (cento e vinte e dois) alunos, o que bem demonstra o interesse da nossa juventude pelo aprendizado em Matemática (IMURN, 1966, p. 1, grifos nossos).

Note-se que, como já afirmamos anteriormente, em 1966, a Matemática Moderna não fazia parte dos currículos dos cursos da Universidade, portanto, a frase ressaltada por nós não se justificaria, naquela época. Tal passagem indica a propagação da idéia que a Matemática Moderna seria "indispensável aos jovens", pois determinaria "o êxito em qualquer curso de nível universitário”, isto é, tal Matemática passou a compor as crenças acerca da inserção do sujeito nas relações de trabalho da época determinadas pela busca do desenvolvimento técnico, relações estas que influenciaram a formação da subjetividade ${ }^{7}$ dos indivíduos de então.

Paralelamente ao CIM, um grupo de professores do Instituto de Matemática ministrou cursos de lógica e de Álgebra Moderna para professores da Escola Secundária, contribuindo, assim, para a inserção do Movimento da Matemática Moderna neste nível escolar no RN.

Também estava prevista, no convênio supracitado entre SUDENE e UFRN, a compra de livros para o IMURN. Foram comprados os seguintes livros:

7 Estamos entendendo "subjetividade" no sentido atribuído por Rosa (1994), ou seja, a subjetividade que se forma nas relações de poder estabelecidas na sociedade e que se relacionam à transformação da pessoa em mercadoria pela venda do trabalho humano que, enquanto mercadoria, é descartável. 


\begin{tabular}{|c|c|c|c|}
\hline Título & Autor & Ano & $\begin{array}{l}\text { Aborda } \\
\text { estruturas } \\
\text { algébricas }\end{array}$ \\
\hline $\begin{array}{l}\text { Éléments de } \\
\text { Mathématique }\end{array}$ & N. Bourbaki & 1959 & $\operatorname{Sim}$ \\
\hline Noveau Cours de & Garnac; E. Ramis; & 1963 & Sim \\
\hline Mathématique Spéciales & J. Comeau & & \\
\hline Modern Álgebra & Seth Warner & 1965 & Sim \\
\hline Elementary Algebra & Barnett Rich & 1960 & Sim \\
\hline Commutative Algebra & $\begin{array}{l}\text { Oscar Zarinski; } \\
\text { Pierre Samuel }\end{array}$ & 1963 & Sim \\
\hline Algebre et Analyse & G. Lefort & 1964 & Sim \\
\hline $\begin{array}{l}\text { L'enseignement de la } \\
\text { Géométrie }\end{array}$ & Gustave Choquet & 1964 & Sim \\
\hline $\begin{array}{l}\text { Theory and Problems of } \\
\text { College Algebra }\end{array}$ & Murray R. Spiegel & 1956 & $\operatorname{Sim}$ \\
\hline $\begin{array}{l}\text { Quadratic Forms and Matrices } \\
\text { An Introductory Approach }\end{array}$ & N. V. Yefimov & 1964 & $\operatorname{Sim}$ \\
\hline The Theory of Groups & A. G. Kurosh & 1960 & Sim \\
\hline Differencial Equations & Frank Ayres Jr. & 1952 & Sim \\
\hline Elements of Number Theory & I.M. Vinogradov & 1954 & Não \\
\hline Cardinal and Ordinal Numbers & Sierpinski & 1965 & Sim \\
\hline Number Theory and its history & Oystein Ore & 1948 & Não \\
\hline Set Theory & Felix Hausdoff & 1962 & Sim \\
\hline Number Theory & J. Hunter & 1964 & Sim \\
\hline $\begin{array}{l}\text { History of The Theory of } \\
\text { Numbers }\end{array}$ & $\begin{array}{l}\text { Leonard Eugene } \\
\text { Dickson }\end{array}$ & 1952 & Não \\
\hline Introduction to field theory & Iain T. Adam Son & 1964 & Sim \\
\hline Elements of Linear Algebra & Lowell j. Paige & 1965 (4. ${ }^{a} \mathrm{ed}$.) & Sim \\
\hline Theory of Equations & H. W. Turnbull & 1963 & Sim \\
\hline Modern Algebra & B. L. Van Der Vaerden & 1964 & Sim \\
\hline $\begin{array}{l}\text { Matrix Algebra for } \\
\text { Electrical Engineers }\end{array}$ & Addison & 1963 & Sim \\
\hline The Theory of Numbers & Hardy and Wright & 1960 & Sim \\
\hline $\begin{array}{l}\text { Introduction to Diferenciable } \\
\text { Manifolds }\end{array}$ & Louis Auslander & $\mathrm{s} / \mathrm{d}$ & $\operatorname{Sim}$ \\
\hline $\begin{array}{l}\text { Theory and Problems of } \\
\text { Modern Algebra }\end{array}$ & Frank Aires Jr. & 1965 & $\operatorname{Sim}$ \\
\hline $\begin{array}{l}\text { Fundamental Concepts of } \\
\text { Algebra }\end{array}$ & Claude Chevalley & 1956 & $\operatorname{Sim}$ \\
\hline $\begin{array}{l}\text { American Mathematical Society } \\
\text { Colloquium Publications }\end{array}$ & Volume X & 1929 & Não \\
\hline Leçons D'Algèbre Moderne & $\begin{array}{l}\text { P. Dubriel ; M. L. } \\
\text { Dubriel ; Jacotin }\end{array}$ & 1964 & $\operatorname{Sim}$ \\
\hline Cours D'Algèbre & Royer Godement & 1966 & Sim \\
\hline Lectures on General Algebra & A. G. Kurosh & 1963 & Sim \\
\hline $\begin{array}{l}\text { Introduction to the Theory } \\
\text { of Finite Groups }\end{array}$ & Walter Lendermann & 1964 & Sim \\
\hline Foundation of Linear Algebra & $\begin{array}{l}\text { W. H. Freeman } \\
\text { and Company }\end{array}$ & 1963 & $\operatorname{Sim}$ \\
\hline
\end{tabular}


É notável a quantidade de livros adquiridos pela verba do convênio entre SUDENE e UFRN que abordam os conceitos matemáticos a partir das estruturas algébricas. A compra destes livros foi orientada pelos professores Antonio Mario Sette, da Universidade Federal de Pernambuco, e Nelson de Almeida Braga, do Instituto de Matemática da Universidade Federal do Ceará.

Deste modo, concluímos que foi por meio daquele convênio que o MMM passou a fazer parte do currículo de matemática da UFRN e, a nosso ver, tal convênio estava relacionado com as estratégias para o desenvolvimento técnico e científico do bloco capitalista.

\section{Referências}

CECINE. Curso de Verão de Matemática (janeiro-fevereiro, 1966). Pernambuco, 1966.

CHAVES FILHO, Q. Relatório apresentado pelo professor Quinho Chaves Filho, por ocasião da transmissão de cargo de Diretor da Faculdade de Educação da UFRN. Natal, 1972.

IMURN. Notícias do Instituto de Matemática. Natal: Universidade do Rio Grande do Norte,1966.

KLINE, M. O fracasso da matemática moderna. São Paulo,SP: IBRASA, 1976. ROSA, M. I. Trabalho, subjetividade e poder. São Paulo: EDUSP, 1994.

SOARES, F. Movimento da matemática moderna no Brasil: avanço ou retrocesso. Dissertação (Mestrado) - PUC RJ, Rio de Janeiro, 2001.

UNIVERSIDADE Do Rio Grande do Norte. Notícias da Escola de Engenharia, Natal. Agosto,1966.

VALENTE, W. Livros didáticos de matemática e as reformas Campos e Capanema. In: ENEM, 8., Recife, 2004. Anais... Recife: SBEM, 2004. 